\title{
Selective removal of reward and nonreward odors to assess their control of patterned responding in rats
}

\author{
RONALD D. TAYLOR and H. WAYNE LUDVIGSON \\ Texas Christian University, Fort Worth, Texas 76129
}

\begin{abstract}
Control of straight-alleyway patterned responding (discrimination) in rats by differential conspecific odors arising from rewarded and nonrewarded trials was examined by selectively removing odors on one or both kinds of trials. Response patterning established with odors undisturbed on both rewarded and nonrewarded trials was maintained when odors were removed on rewarded trials. Removal of odors on both rewarded and nonrewarded trials or on only nonrewarded trials disrupted patterning, although it was reestablished under the latter condition. These data indicate that odors arising both from rewarded and nonrewarded conspecifics can control patterned responding in this task. Although the greater change in speed occurred with removal of "nonreward odor," both odors were functional, as indicated by (1) better patterning based on nonreward vs. clean than based on nonreward vs. reward conditions and (2) a reversal of patterning immediately after removal of nonreward odor only.
\end{abstract}

Previous studies have indicated that laboratory rats excrete odors when receiving typical experimental treatments, such as goal events of reward or nonreward, and that, given appropriate conditions, these odors may serve as discriminative stimuli for other rats (e.g., Ludvigson, 1969; Mellgren, Fouts, \& Martin, 1973; Morrison \& Ludvigson, 1970; Seago, Ludvigson, \& Remley, 1970). One technique used to study this involves patterned running in a straight-alley apparatus. As introduced by Ludvigson and Sytsma (1967), it involves administering reward and nonreward to a squad of rats, with each animal receiving the same goal event on a given trial, and administering trials to the animals in rotation such that all animals receive the first trial before any animal receives the second, and so on. If odor-removal procedures are restricted to the beginning of a trial, thus permitting any excreted odors to remain in the apparatus as all animals receive a given trial, what emerges is a pattern of fast running on rewarded trials and slow running on nonrewarded trials, so long as the response is measured toward the goal end of the apparatus. This response patterning is largely, if not exclusively, the result of differential conditioning controlled by discriminatively different odors.

Although studies using the patterned responding design have rather clearly established that there is some

Portions of this research were presented at the meeting of the Southwestern Psychological Association, Fort Worth, Texas, 1977. Funding for the research was provided in part by a fellowship awarded to the first author by the Chemistry of Behavior Program and by the Texas Christian University Research Foundation. Requests for reprints should be sent to Ronald D. Taylor, Department of Psychology, Salem College, Salem, West Virginia 26426. difference between odor emissions from rewarded as compared with nonrewarded trials, they do not permit a specification of the nature of the difference. Thus, it is not clear whether the discriminative odors arise from nonrewarded trials, rewarded trials, or both. Quite a number of studies employing various designs suggest that odor arising from nonrewarded or frustration trials must certainly be one of the discriminative stimuli (Collerain, 1978; Collerain \& Ludvigson, 1972, 1977; Ludvigson, McNeese, \& Collerain, 1979; Mellgren et al., 1973; Morrison \& Ludvigson, 1970). In addition, studies utilizing sodium amobarbital (Howard \& McHose, 1974) and Thorazine (Davis \& Prytula, 1979) found that administering these drugs to donor subjects eliminated response patterning in test subjects that followed them, presumably because the drug prevented the donor subjects from becoming emotionally aroused under conditions of frustration. Of course, the drug could also have interfered with emotional arousal on rewarded trials, although the evidence justifying an inference of emotionality there is not so strong. More critically, recent evidence points to the existence of an odor on rewarded trials (Collerain \& Ludvigson, 1977; Mellgren et al., 1973), indicating that the patterned running effect could be controlled by odors arising on rewarded trials as well as on nonrewarded trials.

This experiment involved a direct examination of the roles of reward and frustration odors in patterned responding ${ }^{1}$ through the technique of selective removal of odors on one kind or both kinds of trials. Thus, after response patterning was clearly established, the effects of removing odors resulting from rewarded trials and the effects of removing odors from nonrewarded trials were systematically evaluated. 


\section{METHOD}

Subjects

The subjects were seven male albino rats obtained from the Holtzman Company. They were 193 days of age on Day 1 . The rats had previously received 544 trials of $50 \%$ partial reinforcement in the straight alley used herein while serving as odor-donor subjects in another experiment. They were maintained at approximately $85 \%$ of their preexperimental ad-lib weights throughout the experiment.

\section{Apparatus}

A straight alley $221 \mathrm{~cm}$ in length, $7.62 \mathrm{~cm}$ wide, and $8.89 \mathrm{~cm}$ high served as the experimental apparatus. The alleyway was divided into a $30-\mathrm{cm}$ startbox, a $114-\mathrm{cm}$ run section, and a $59-\mathrm{cm}$ goalbox. The apparatus was constructed of wood, painted white, and had a hinged Plexiglas top. A startdoor microswitch and an arrangement of photocells located 15, 75, 136, and $166 \mathrm{~cm}$ from the startdoor allowed the measurement of a start time, two run times, and a goal time. A glass food cup, painted white, was present at the end of the goalbox on all trials, and an externally mounted pellet dispenser was used to dispense $45-\mathrm{mg}$ Noyes pellets through the end of the goalbox and into the food cup on rewarded trials. Adding machine paper covered the alley floor, coming from a roll affixed outside the start end of the alley so as to permit the paper to be pulled through a slot in the floor at the goal end.

Cleaning of the alley was accomplished by a complete replacement of the paper flooring and air within the alley. The air replacement was accomplished by activating a standard vacuum cleaner, attached at the goal end, which pulled air from the experimental room through open ports in the startbox end and removed air through the vacuum cleaner hose to an airreturn duct leading to a central temperature-control unit.

\section{Procedure}

The daily schedule of reward and nonreward was determined by progression through a repeated 4-day cycle of RNNRRRNN, RRNNNRRN, NRRNNNRR, and NNRRRNNR, where R and $N$ represent reward and nonreward, respectively. The order in which the rats were run was constant on all trials, and all animals received the first trial before any animal received the second, and so on. Before each trial, the alleyway was cleaned by changing the paper flooring and exhausting the air. On rewarded trials, two $45-\mathrm{mg}$ Noyes pellets were present in the food cup and 18 more were dispensed after the animal interrupted the last photobeam. A stopwatch was started when the animal entered the goalbox, and $30 \mathrm{sec}$ was allowed for food consumption on rewarded trials and for confinement to the unbaited goalbox on nonrewarded trials.

On Days 1 and 2, the alleyway was left undisturbed on both rewarded and nonrewarded trials. This condition, the $\mathrm{R}+\mathrm{N}+$ condition, represents a baseline for the experimental treatments. The first treatment, on Days 3-5, involved cleaning the alley after each animal on both rewarded and nonrewarded trials. This $\mathrm{R}-\mathrm{N}$ - condition was a control to assess the effectiveness of the odor-removal procedures. On Day 3 the alley air was exhausted for $20 \mathrm{sec}$; on all subsequent days, the air was exhausted for $60 \mathrm{sec}$.

Days 6-7 represented a return to baseline in preparation for treatments on Days 8-10. On Days 8-10 the alley was cleaned after each animal on rewarded trials but was left undisturbed on nonrewarded trials, a condition referred to as the $\mathrm{R}-\mathrm{N}+$ treatment. Days 11-12 represented a return to baseline discrimination. Days $13-20$, the $\mathrm{R}+\mathrm{N}-$ treatment, involved cleaning the alleyway after each animal on nonrewarded trials and leaving the alleyway undisturbed on rewarded trials. On Days 21-26 there was another test to assess the extent to which odor-removal procedures were effective in eliminating the discrimination. On Days 24-26, a hand-operated vacuum cleaner with brush nozzle was used to clean the sides and top of the goalbox, in addition to exhausting the air and changing the paper in the alley as before.

An intersubject interval of 3 min was maintained throughout the experiment. On all trials, each animal was carried in its home cage from a closed room to the experimental room and then was taken to a third room following removal from the goalbox. A white-noise generator provided a constant background noise of about $85 \mathrm{~dB}$ in the experimental room throughout the experiment.

\section{RESULTS}

Each subject's mean daily goal speed was computed for rewarded and for nonrewarded trials, and the group means for Subjects 2-7 (i.e., excluding the first subject in the squad, since discriminative odor cues should not have been present for this subject) supplied the data for Figure 1. In Figure 1, treatments are represented at the top by the letters $\mathrm{R}$ and $\mathrm{N}$, each followed by either a plus or minus sign, indicating that odors were left undisturbed or removed, respectively.

Panel A of Figure 1 simply shows 2 days of characteristic patterned responding, fast on rewarded trials and slow on nonrewarded trials, established by the previous 544 trials, during which odors were undisturbed on both rewarded and nonrewarded trials.

\section{First Test of Odor-Removal Procedures}

The first treatment, represented in Panel B of Figure 1 , involved removing odors on both rewarded and nonrewarded trials as a test of the effectiveness of odorremoval procedures. A $t$ test for related means performed on the goal speeds for Day 3 was not significant; however, as may be seen in Figure 1, there was some suggestion that odor-removal procedures were not completely effective on Day 3. Therefore, the time during which the alley air was exhausted was increased

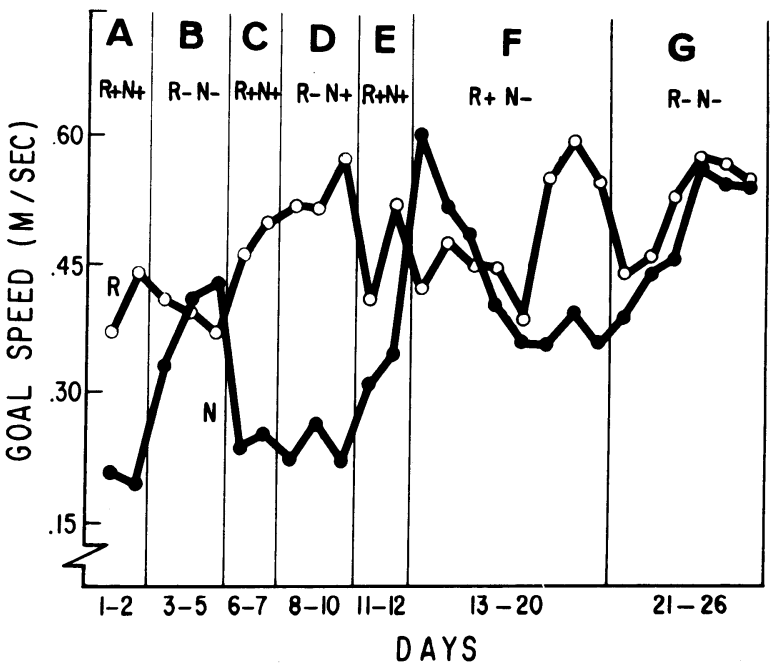

Figure 1. Mean daily goal speeds for rewarded (R) and nonrewarded $(\mathrm{N})$ trials as a function of selective odor removal. $(+=$ paper flooring and alley air left undisturbed; $-=$ paper flooring and alley air replaced with clean.) 
from $20 \mathrm{sec}$ on Day 3 to $60 \mathrm{sec}$ on all subsequent days. The data were placed into a randomized-block factorial design and submitted to an analysis of variance with repeated measures on treatments (Phase A vs. Phase B) and goal events ( $R$ vs. $N$ ). The analysis produced significant effects for goal events $[F(1,15)=19.3, p<.001]$, treatments $[\mathrm{F}(1,15)=19.52, \mathrm{p}<.001]$, and the Goal Events by Treatments interaction $[F(1,15)=33.67$, $\mathrm{p}<.001]$. A follow-up on the interaction indicated that goal speeds on nonrewarded trials increased significantly from Phase A to Phase B $[F(1,15)=52.85$, $\mathrm{p}<.01]$, but they did not change significantly on rewarded trials. Furthermore, although a significant discrimination was present in Phase $\mathrm{A}[\mathrm{F}(1,15)=52.58$, $\mathrm{p}<.01$ ], it was eliminated in Phase B. This indicates that the odor-removal procedures were effective (Phase B) and that most, if not all, of the disruption was due to the removal of frustration odor.

Removal of $\mathbf{R}$ odors. Panels $\mathbf{C}$ and $\mathrm{E}$ of Figure 1 represent the baseline discrimination, and Panel $\mathrm{D}$ represents the level of discrimination when odors were removed on rewarded trials and left undisturbed on nonrewarded trials. For purposes of statistical analysis, each subject's goal speeds in Phases $\mathrm{C}$ and $\mathrm{E}$ were averaged. Analysis of variance with repeated measures on treatments (Phases $\mathrm{C}$ and $\mathrm{E}$ vs. Phase D) and goal events ( $R$ vs. N) produced a significant effect for goal events $[F(1,15)=194.00, p<.01]$ and a significant Goal Events by Treatments interaction $[F(1,15)=11.00$, $\mathrm{p}<.01]$. Analysis of simple main effects showed faster goal speeds on rewarded trials than on nonrewarded trials both in Phases $\mathrm{C}$ and $\mathrm{E}[\mathrm{F}(1,15)=55.50, \mathrm{p}<.01]$ and in Phase $\mathrm{D}[\mathrm{F}(1,15)=149.95, \mathrm{p}<.01]$. In addition, goal speeds on rewarded trials were faster during Phase $\mathrm{D}$ than during Phases $C$ and $E[F(1,15)=7.00, p<.05]$, although no significant differences appeared for nonrewarded trials across phases. Hence, the subjects showed patterned responding throughout Phases C, D, and E, but they actually ran faster on rewarded trials when reward odor was removed than when it was not removed.

Removal of $\mathbf{N}$ odors. Panel $\mathrm{F}$ of Figure 1 shows that when frustration odor was removed while reward odor was undisturbed, the response patterning reversed itself, with subjects actually running faster in the goal area on nonrewarded trials than on rewarded trials $[\mathrm{t}(5)=3.64$, $\mathrm{p}<.05]$. However, by the end of Phase F, Days 19 and 20 , the subjects were again running faster on rewarded trials than on nonrewarded trials $[\mathrm{t}(5)=4.99, \mathrm{p}<.01]$, indicating further that reward odor was indeed present and discriminably different from a clean alley.

\section{Second Test of Odor-Removal Procedures}

The last phase was a test to assess the extent to which the odor-removal procedures were still effective in controlling the discrimination. On Days 21-23 (Figure 1, Panel G) a small discrimination remained, despite the exchange of alley air and paper on both rewarded and nonrewarded trials. Consequently, on Days 24-26, not only was the alley air exhausted and paper exchanged, but also the walls and ceiling of the goalbox were vacuumed with a brush nozzle. An analysis of variance with repeated measures on days (21-23 vs. $24-26)$ and goal events ( $R$ vs. $N$ ) produced a significant effect of goal events $[F(1,15)=7.14, p<.05]$ and a significant effect of days $[F(1,15)=101.43, p<.01]$, but no interaction and thus no indication that the additional cleaning was effective.

\section{Other Measures}

Data from other sections of the alleyway are not presented because they add little to the results reflected in goal speeds. The discrimination would not be expected to occur in the initial portions of the alleyway, since the discriminative stimuli occur at the goal end. An examination of start speeds and run speeds in the first half of the alley gave no indication of patterned responding, whereas run speeds in the last half of the alley, just preceding the goalbox, generally reflected the differences observed in the goal measure.

\section{DISCUSSION}

This study provides further support for the hypothesis that laboratory rats excrete differential odors as a function of both rewarded and nonrewarded experiences. The dramatic increase in the animals' speeds on $\mathrm{N}$ - trials at the outset of Phase $\mathrm{F}$ $(\mathrm{R}+\mathrm{N}-)$ suggests that the typical response patterning in the $\mathrm{R}+\mathrm{N}+$ condition is strongly under the control of frustration odor. Support for this inference comes from the observation that the transition from Phase A to Phase B was accompanied by a sharp increase in speeds on nonrewarded trials and relatively little change on rewarded trials, despite the fact that these subjects had never encountered a clean goalbox before this treatment. However, the fact that patterning actually reversed itself on Day 1 of Phase F is evidence that it is not the case that reward odor is simply nonfunctional. Had it been nonfunctional, speeds on $\mathrm{N}$ trials should have equaled, but not exceeded, speeds on $\mathrm{R}$ trials.

The animals' performance in Phase $D(R-N+)$ as compared with Phases $\mathrm{C}$ and $\mathrm{E}(\mathrm{R}+\mathrm{N}+)$ suggests that the discrimination is easier when reward odor is removed. That is, discriminating frustration odor from a clean alley is easier than discriminating frustration odor from reward odor. This lends weight to the conclusion that reward odor does affect responding in this task. In addition, it suggests that reward and frustration odors are psychologically more similar than are frustration odor and a clean alley.

Some caution should be exercised in interpreting the discrimination that eventually developed during the $\mathrm{R}+\mathrm{N}-$ phase. The failure to eliminate completely the discrimination in the last phase of the study suggest either that the odor-removal procedures were no longer completely effective at that point or that something else, such as the odor from the two food pellets awaiting the subject on rewarded trials was partially controlling the discrimination at this point in the study. However, it is clear that the discrimination in the $\mathrm{R}+\mathrm{N}-$ phase was predominantly controlled by odor left by the preceding subjects on rewarded trials: The treatment $(\mathrm{R}-\mathrm{N}-)$ that subsequently specifically removed odor, or at least a good part of it, sharply reduced discrimination (Phase $G$ ).

While this study suggests that both reward and frustration odors are present and functional, it does not necessarily implicate two odors uniquely produced by different (classes of ) operations, since, for example, one could be characteristic of the animals 
regardless of operations. Alternatively, the odors might be different concentrations of the same emission. Nevertheless, the present data are not inconsistent with studies that suggest two odors distinct in some property from the rats' characteristic scent (Cattarelli, Vernet-Maury, \& Chanel, 1974, 1977² Collerain \& Ludvigson, 1977; Mellgren et al., 1973).

\section{REFERENCES}

Cattarelli, M., Vernet-Maury, E., \& Chanel, J. Influences de differentés odeurs biologiques sur le comportement émotif du rat placé dan un "espace vide d'informations." Comptes Rendus de l'Académie des Sciences de Paris, 1974, 278, 2653-2656. (Série D)

Cattarelli, M., Vernet-Maury, E., \& Chanel, J. Modulation de l'activité due bulbe offactif en fonction de la signification des odeurs chez le rat. Physiology \& Behavior, 1977, 19, 381-387.

Collerain, I. Frustration odor of rats receiving small numbers of prior rewarded running trials. Journal of Experimental Psychology: Animal Behavior Processes, 1978, 4, 120-130.

Collerain, I., \& Ludvigson, H. W. Aversion of conspecific odor of frustrative nonreward in rats. Psychonomic Science, 1972, 27, 54-56.

Collerain, I., \& Ludvigson, H. W. Hurdle-jump responding in the rat as a function of conspecific odor of reward and nonreward. Animal Learning \& Behavior, 1977, 5, 177-183.

Davis, S. F., \& PRYTulA, R. E. Odor-mediated runway performance of the rat as a function of Thorazine injection. Bulletin of the Psychonomic Society, 1979, 13, 293-296.

Howard, G. S., \& McHose, J. H. The effects of sodium amobarbital on odor-based responding in rats. Bulletin of the Psychonomic Society, 1974, 3, 185-186.

Ludvigson, H. W. Runway behavior of the rat as a function of intersubject reward contingencies and constancy of daily reward schedule. Psychonomic Science, 1969, 15, 41-43.
Ludvigson, H. W., McNeese, R., \& Collerain, I. Long-term reaction of the rat to conspecific (frustration) odor. Animal Learning \& Behavior, 1979, 7, 251-258.

Ludvigson, H. W., \& Sytsma, D. The sweet smell of success: Apparent double alternation in the rat. Psychonomic Science, 1967, 9, 283-284.

Mellgren, R. L., Fouts, R. S., \& Martin, J. W. Approach and escape to conspecific odors of reward and nonreward in rats. Animal Learning \& Behavior, 1973, 1, 129-132.

Morrison, R. R., \& Ludvigson, H. W. Discrimination by rats of conspecific odors of reward and nonreward. Science, 1970, 167, 904-905.

Seago, J. D., Ludvigson, H. W., \& Remley, N. R. Effects of anosmia on apparent double alternation in the rat. Journal of Comparative and Physiological Psychology, 1970, 71, 435-442.

\section{NOTES}

1. The reader should note that we use the terms "frustration odor," "reward odor," and the like as convenient names for the discriminably different odors resulting from different treatments. No prejudice is intended with regard to the question of the chemical and/or psychological uniqueness of the odors, or even of the size of the class of treatments producing odors.

2. From personal communication, it appears their operational definition of "frustration" involves, at least sometimes, what might be termed "fear" and "conflict"; that is, in addition to cues previously associated with reward, cues previously associated with electric shock are presented that deter the animal from obtaining the reward.

(Received for publication June 12, 1980.) 